\title{
Influence of External Current on Yokeless Electric Current Transducers
}

\author{
Pavel Ripka, Andrey Chirtsov \\ Czech Technical University, Faculty of Electrical Engineering \\ Prague, Czech Republic
}

\begin{abstract}
Yokeless electric current transducers have compact size, but the y are sensitive to external magnetic fields including those caused by electric currents in their vicinity. It is often believed that this unwanted sensitivity can be effectively suppressed by using differential sensor. In this paper we in vestigate the effect of external current with arbitrary position on busbar differential current sensor. We show the main disadvan tage of differential current sensor: increased sensitivity to currents in trans versal direction, which are not sensed by a single sensor. We analyze by FEL simulation also the influence of real conductor size and uneven density of AC currents. The results we re verified on 1000 A current transducer using a pair of microfluxgate sensors. The realistic suppression of close currents de pends on the conductor angular position and in $10 \mathrm{~cm}$ distance it can be as low as 50 , but it can be corrected if the geometry is known.
\end{abstract}

Index Terms-current sensor, fluxgate, Hall sensor, microfluxgate

\section{INTRODUCTION}

Contag ontactless transducers of DC electric currents often have magnetic yoke, which concentrates the flux generated by the measured current. As a result, the reading does not depend much on the conductor position inside the magnetic circuit. The yoke also shields against external magnetic fields including those caused by external currents (crosstalk error) [1]. Hall sensors, which are slimin the measuring direction, fit into the narrow airgap in the yoke and therefore dominate in this application. Other transducers of this type have small fluxgate sens or in the slot of the yoke, or the whole yoke is AC excited and works as fluxgate sensor [2,3]. Closed-loop current transducers with yoke easily achieve accuracy below $0.1 \%$. However, for many applications theopen-loop configuration is used, which is less power consuming and low-cost. Sensitivity drift of the Hall sensor can be compensated using microsystem with autocalibration coil [4] down to $80 \mathrm{ppm} / \mathrm{K}$.

However for large currents and high-voltage networks the yoke becomes too large and heavy to prevent saturation and ensure the required distance fromthe high-voltage conductor. In these cases the yokeless solution is required. The important advantage of yokeless current sensor is the absence of ferromagnetic material which can be saturated by overcurrent [5]. The integrated yokeless current sensors have limited current range: 5 A sensor of this type is described in [6]. Commercially-available yokeless high current transducers use discrete sensors on both sides of the bus bar [7]. This configuration has principle disadvantages:

1. magnetic field on the surface of the conductor is large so that precise magnetic sensors such as magnetoresis tors or fluxgates cannot be used.

2. busbar movement or uneven current distribution in the bus bar cau ses meas urement error

3. Suppression of the external currents by gradiometric sensor

Manuscript received April 1, 2015; revised May 15, 2015 and June 1, 2015; accepted July 1, 2015. Date of publication July 10, 2015; date of current version July 31, 2015. Corresponding author: P. Ripka (e-mail: ripka@fel.cvut.cz. Color versions of one or more of the figures in this paper are available online at http://ieexplore.ieee.org.

Digital Object Identifier (inserted by IEEE). is low as the sensor distanceis high. Later in this paperwe derive formula proving this claimfor arbitrary position of the external conductor.

George suggested to suppress the influence of the meas ured conductor position by calculating $\mathrm{B}_{1} * \mathrm{~B}_{2} /\left(\mathrm{B}_{1}+\mathrm{B}_{2}\right)$ [8]. However this trick would destroy the immunity of the differential transducer ag ainst external currents.

Chen used four Hall sensors on each conductor and measured errors caused by external currents [9]. He found that the difference between calculated and measured error was below $6 \%$ for conductors in the close vicinity and this difference is rapidly decreasing with increasing distance between conductors.

Circular sensor array with more than four sensors around the conductor approximates better the closed line integral in the Amper's law. This brings better immunity against the position of the measured conductor [10] as well as against the crosstalk from external conductors [11]. Both errors decrease with increasing number of sensors, 8 sensors are considered as an optimum number. Further error reduction can be achieved by using crosstalk reduction algorithm, but this is impractical for industrial applications due to the large computational complexity of the necessary non-linear solver. Circular sensor array still limits the maximum measurable current when using precise magnetic sensors. For $100 \mathrm{~mm}$ diameter array the maximum measurable current is $50 \mathrm{~A}$ for $\mathrm{AMR}$ and $500 \mathrm{~A}$ for microfluxgate sensor, taking into account their field range of $0,2 \mathrm{mT}$ and $2 \mathrm{mT}$ respectively.

In this paper we analyze the influence of external current on the differential current transducer. We start with analytical calculation of the influence of the idealized current, follow with FEL simulation of the real conductor including the effects of the eddy currents and finish by experimental verification of selected configurations.

\section{BUSSBAR TRANSDUCER FOR HIGH CURRENTS}

Mounting a differential magnetic field sensor inside the busbar solves the problem with limited range as the sensitivity s and thus the current range can be adjusted by the sensor distance 
from the center of symmetry [12]. External currents are suppressed by measuring the field gradient using a pair of sensors.

For all measurements in this paper we have used DRV425 microfluxgate sensors [13]. These sensors have very low offset tempco of $5 \mathrm{nT} / \mathrm{K}$ which makes them superior to other roomtemperature miniature magnetic sensors such as Hall $(5 \mu \mathrm{T} / \mathrm{K}$ is typical value for commercially available devices such as Infenion TLE4997) or any type of magnetoresistors ( $241 \mathrm{nT} / \mathrm{K}$ for KMZ 51). They als o have low crossfield error below $10 \mathrm{nT}$ [14] which results in excellent linearity for the uncompensated sensor.

In our case the differential microfluxgate sensors is inserted into the cylindrical $19 \mathrm{~mm}$ hole in the $60^{*} 10 \mathrm{~mm}$ copper busbar. For the sensor distance of $2 \mathrm{a}=2,5 \mathrm{~mm}$ the sensitivity to the $\mathrm{DC}$ measured current calculated by FEM is $\mathrm{s}=2(\mathrm{~A} / \mathrm{m}) / \mathrm{A}$ and this value was also verified experimentally. For AC currents the current density is no longer homogeneous due to the eddy currents and the sensitivity drops down with frequency. Fig. 1 shows the calculated sensitivity as a function of sensor distance for DC measured current and also for ACmeasured current with $\mathrm{f}=1 \mathrm{kHz}$. Decreasing sensor distance generally reduces crosstalk error, but the effective suppression factor is even getting worse due to the decreased sensitivity s to the measured field.

\section{FIG. 1 HERE}

\section{LATERAL EXTERNAL CURRENT}

Parasitic response to the external current can be analytically calculated only for the simplified case when the current is localized to one point. For the differential sensor with spacing (base) of $2 \mathrm{a}$ the parasitic response to the idealized external current $I$ in the distance of $d$ in the same plane is

(1)

$$
H_{1}-H_{3}=I \frac{a}{\pi(d+2 a) d}
$$

Response to the realis tic external in-plane current bar with DC current of 100 A was modelled by FEM. The result of such simulation for a single sensor is shown in Fig. 1a) and for differential sensor in Fig. 1 b). The simulated values for differential sensor are also compared to the measured values and values calculated using (1).

For our selected geometry the external current in a $9 \mathrm{~cm}$ distant busbar is suppressed only by the factor of 66 . Compared to the circular array of 8 sensors, which for the same distance has a suppression of 250 , the crosstalk error is still high.

\section{SUPERIOR EXTERNAL CURRENT}

The less known fact is that if the external current is outside the plane, the situation is not much better. If the external current I is in the perpendicular plane, the gradiometer does notsuppress it any more. The simplified situation is illustrated in Fig. 3: sensors 1 and 3 measure field fromtwo halves of busbar sensor current $\mathrm{I}_{\mathrm{m}}$. This current creates field components $\mathrm{H}_{1 \mathrm{~m}}$ and $\mathrm{H}_{3 \mathrm{~m}}$ in the sensitive axes of the two sensors. The idealized localized external field $\mathrm{I}$ in the distance $\mathrm{d}$ creates fields $\mathrm{H}_{1}$ and $\mathrm{H}_{3}$ from which the sensors meas ure their $\mathrm{H}_{1 y}$ and $\mathrm{H}_{3 y}$ components. The parasitic response is very similar to the previous case:

(2)

$$
H_{1 y}-H_{3 y}=I \frac{a}{\pi d^{2}}
$$

\section{FIG. 3 HERE}

\section{A. Real conductors: DC case}

For the real conductors the current is homogeneously distributed in the external busbar and the field map is shown in Fig. 4.

\section{FIG. 4 HERE}

The parasitic response for DC superior current is shown in Fig. 5 . The shape of the characteristics in the close vicinity of the busbars is complicated, but for practical applications this case is not realistic. For larger distances the characteristics approximate the analytically calculated monotonous dependence.

\section{FIG. 5 HERE}

\section{B. The AC case}

If the external current is alternating, the situation is complicated by the influence of the eddy currents: the current density in the external busbar is not uniform and external currentals o induces eddy currents in the measured conductor. Fig. 6 shows an example of current and field dis tribution for external current of $100 \mathrm{~A} / 1 \mathrm{kHz}$. However, the FEM calculated response in larger distances is not different from the DC case.

\section{FIG. 6 HERE}

\section{EXTERNAL CURRENT IN ARBITRARY POSITION}

Fig. 7a illus trates the general case, when the external field I is declined by an angle phi from the sensor line. The field was 
calculated for $\mathrm{d}=0,1 \mathrm{~m}, \mathrm{I}=100 \mathrm{~A}$ and $\mathrm{a}=0,00125 \mathrm{~m}$. The field difference caused by the idealized external current I can be again easily calculated analytically:

(3)

where

$$
\left.H_{1 y}-H_{3 y}=I\left(\frac{\cos \varphi_{1}}{r_{1}}-\frac{\cos \varphi_{3}}{r_{3}}\right)\right)
$$$$
\begin{aligned}
& \varphi_{1}=\operatorname{arctg} \frac{r \cdot \sin \varphi}{r \cdot \cos \varphi+a} \quad \varphi_{3}=\operatorname{arctg} \frac{r \cdot \sin \varphi}{r \cdot \cos \varphi-a} \\
& r_{1,3}=\frac{r \sin \varphi}{\sin \varphi_{1,3}}
\end{aligned}
$$

The calculated values from (3) are shown in Fig. $7 b$ and the result of simulations together with the measured response are shown in Fig. 8. It is clear that (3) can be used only for large distances between the busbars.

\section{FIG. 7 HERE}

\section{FIG. 8 HERE}

\section{CONCLUSION}

External current has significant influence on the reading of the yokeless current tran sducer. Circular trans ducers with typically 8 sensors present the solution with the best crosscurrent suppression. However for $10 \mathrm{~cm}$ diameter their range is limited by the Amper's law to $50 \mathrm{~A}$ for AMR and $500 \mathrm{~A}$ for microfluxgate sens ors. Hall sens ors can be used to increase the measuring range, but only for AC currents, as they DC drift is 1000-times higher compared to microfluxgate sensors.

A transducer with differential fluxgate sensor inside the busbar can overcome this limitation, however its sensitivity to extemal currents is high. We show that this unwanted sensitivity depends on the angular position in more complex way then it was generally believed. The response reaches minimum for the angle of 45 degree, and for larger angles starts again increase. The res ponse to external currents depends on frequency only in very small distances.

If the position of the external conductor is fixed (such as in three-phase systems or s witchboards) and all the currents are measured, compensation of the cross-sensitivity can be calculated based on the calculated cross-sensitivity parameters.

\section{ACKNOWLEDGMENT}

This work was supported by the Grant agency of the Czech Republic through the project "New methods for the measurement of electric currents" (GACR 17-19877S). A. Chirtsov was also supported by Texas Instruments.

\section{REFERENCES}

[1] P. Ripka, Electric current sensors: a review, Meas. Science and Technology 21 (2010) Iss. 11, pp.1-23.

[2] Ripka, P. - Draxler, K. - Styblíková, R.: AC/DC Current Transformer with Single Winding IEEE Trans Magn. 50 (2014) Article \#: 8400504 pp. 1-4,
[3] Xiaoguang Yang; Yuanyuan Li; Weidong Zheng; Wei Guo; Youhua Wang; Rongge Yan Design and Realization of a Novel Compact Fluxgate Current Sensor, Magnetics, IEEE Trans. 51 (2015), Article \#: 4002804

[4] Ajbl, A.; Pastre, M.; Kayal, M. A fully integrated Hall sensor microsystem for contactless current measurement. IEEE Sensors J. 13 (2013), 22712278, 10.1109/JSEN.2013.2251971.

[5] Yuan-Pin Tsai, Kun-Long Chen, Yan-Ru Chen, and Nanming Chen: Multifunctional Coreless Hall-Effect Current Transformer for the Protection and Measurement of Power Systems, IEEE Trans. Magn 63 (2014), 557-565.

[6] Frick, V.; Hebrard, L.; Poure, P.; Anstotz, F.; Braun, F. CMOS microsystem for AC current measurement with galvanic isolation. IEEE Sensors J. 3 (2003), 752-760

[7] M. Blagojevič, U. Jovanovič, I. Jovanovič, D. Mančič, and R. S. Popovič, "Realization and optimization of bus bar current transducers based on Hall effect sensors," Meas. Sci. Technol. 27 (2016), p. 065102.

[8] N. George and S. Gopalakrishna, "An improved anti-differential configuration based hall-effect current sensor," 2016 IEEE Annual India Conference (INDICON), Bangalore, 2016, pp. 1-5.

[9] Chen K.L.; Chen, N. A new method for power current measurement using a coreless Hall effect current transformer. IEEE Trans. Instrum. Meas. 60 (2011), 158-169.

[10] P. Mlejnek, M. Vopalensky, P. Ripka, "AMR current measurement device", Sensors and Actuators A, 141 (2008), pp. 649-653.

[11] L. Di Rienzo, Z. Zhang, "Spatial harmonic expansion for use with magnetic sensor arrays", IEEE Trans. Magn., 46 (2010), pp. 53-58.

[12] P.Ripka, M.Přibil, V. Petrucha, V. Grim, and K.Draxler: A fluxgate current sensor with an amphitheater busbar, IEEE Trans. Magn. 52 (2016), paper \# 4002004

[13] M. F. Snoeij, V. Schaffer, S. Udayashankar, Mikhail V. Ivanov: An Integrated Fluxgate Magnetometer for use in Closed-Loop/Open-Loop Isolated Current Sensing, IEEE J. Solid-state circuits 51 (2016), 16841694.

[14] P. Ripka, M. Janosek, M. Butta, S. W. Billingsley, E. Wakefield: Crossfield error in fluxgate and AMR sensors, Journal of Electrical Engineering. 61 (2010), p. 13-16. 


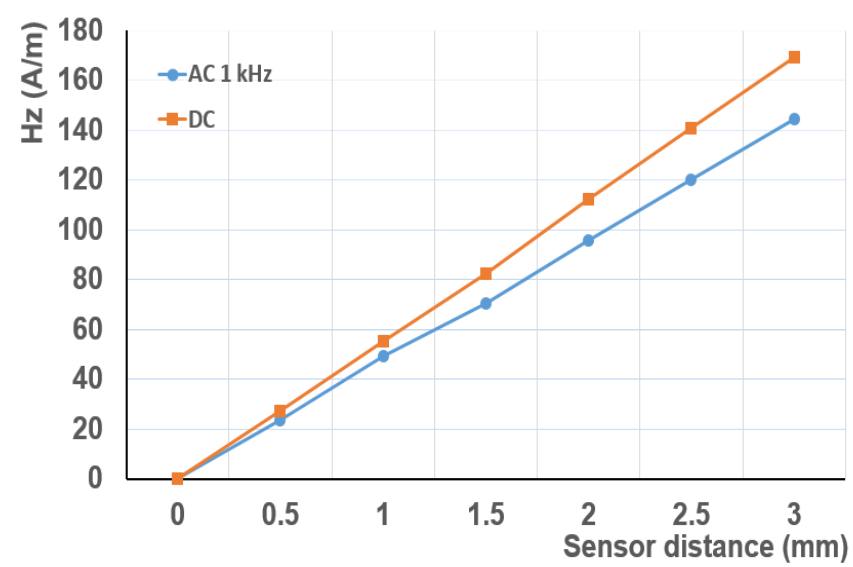

Fig. 1. Sensitivity s as a function of the sensor gradiometric distance 2a (FEM simulation) for DC and $1 \mathrm{kHz}$
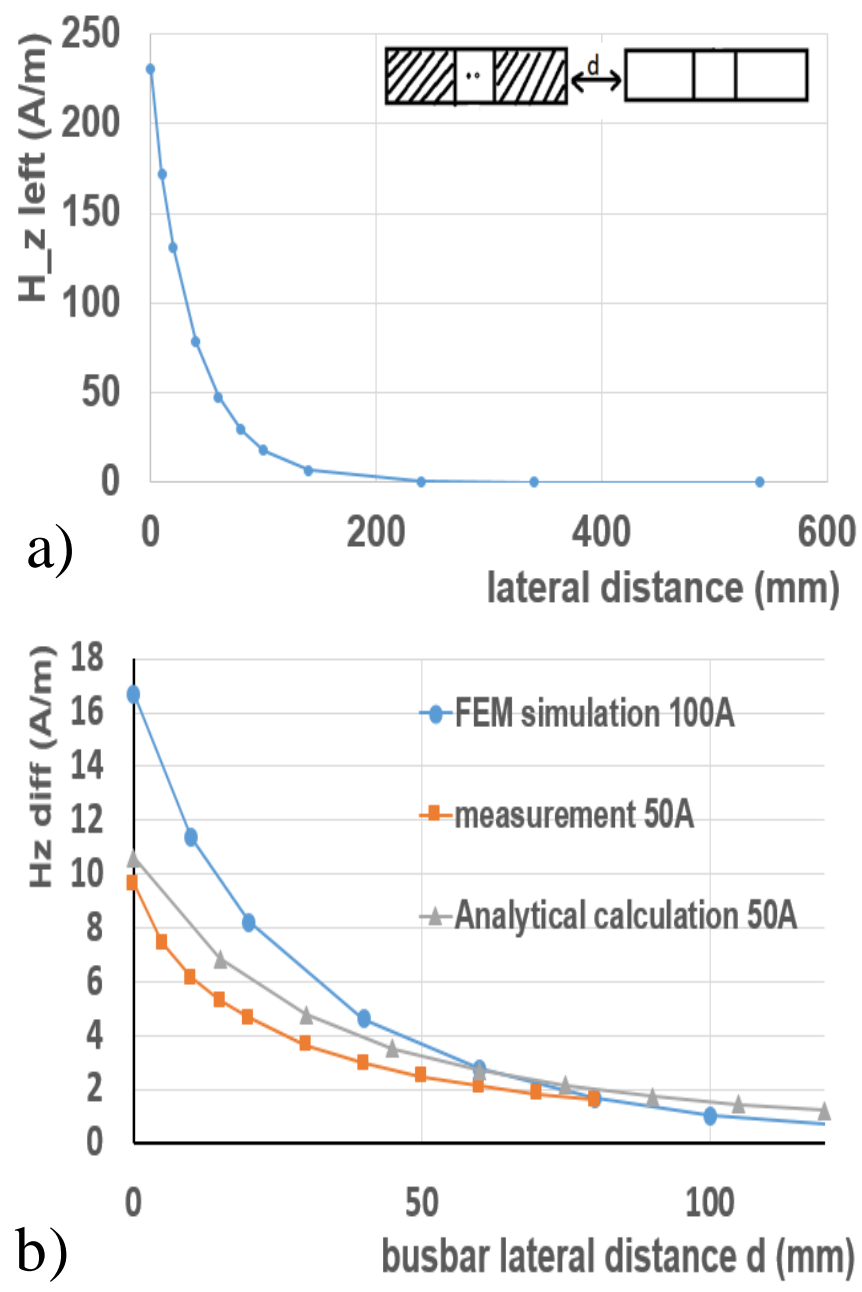

Fig. 2. Response to external lateral $100 \mathrm{ADC}$ current as a function of distance between the busbars: a) FEM calculated single sensor b) differential sensor: upper trace: FEM calculated simulation for $100 \mathrm{~A}$ DC current, middle trace: analytical calculation, lower trace: measurement for $50 \mathrm{~A}$ current

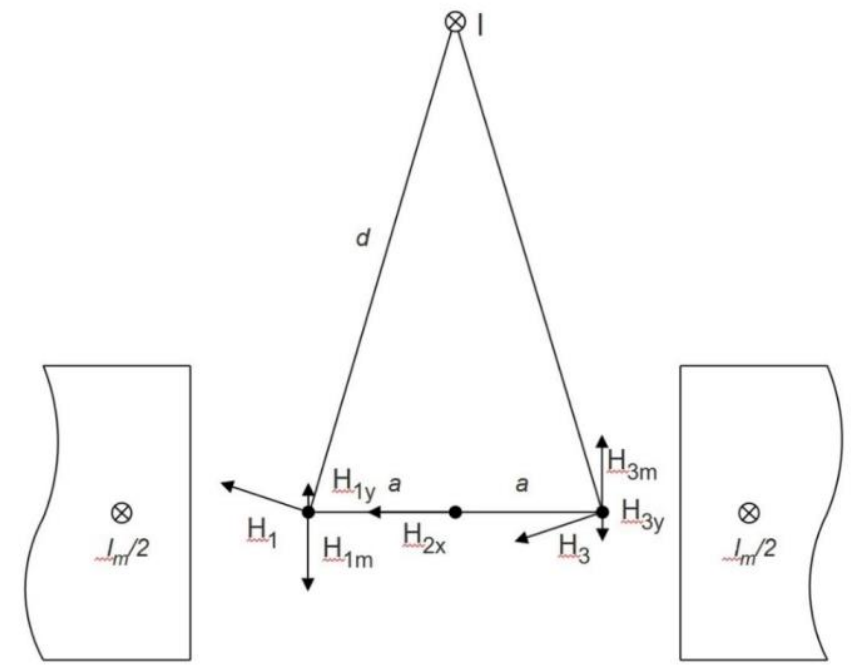

Fig. 3. The magnetic field components in points 1 and 2 where the two sensors are located. These sensors measure field in y direction.

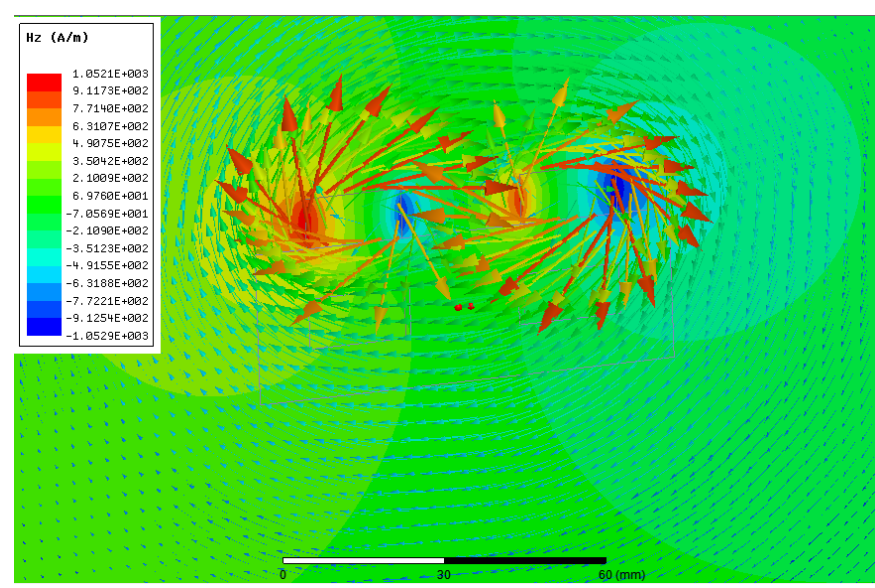

Fig. 4. Field vector image for external busbar in superior position in $10 \mathrm{~mm}$ distance from the measuring busbar. 


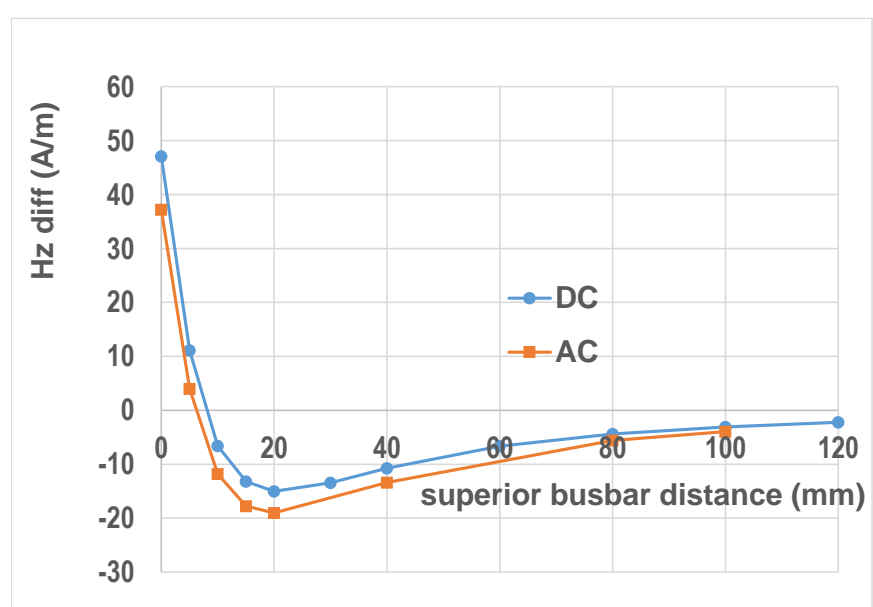

Fig. 5. Response to external superior $100 \mathrm{~A}$ DC current as a function of a distance between the busbars (FEM simulation)

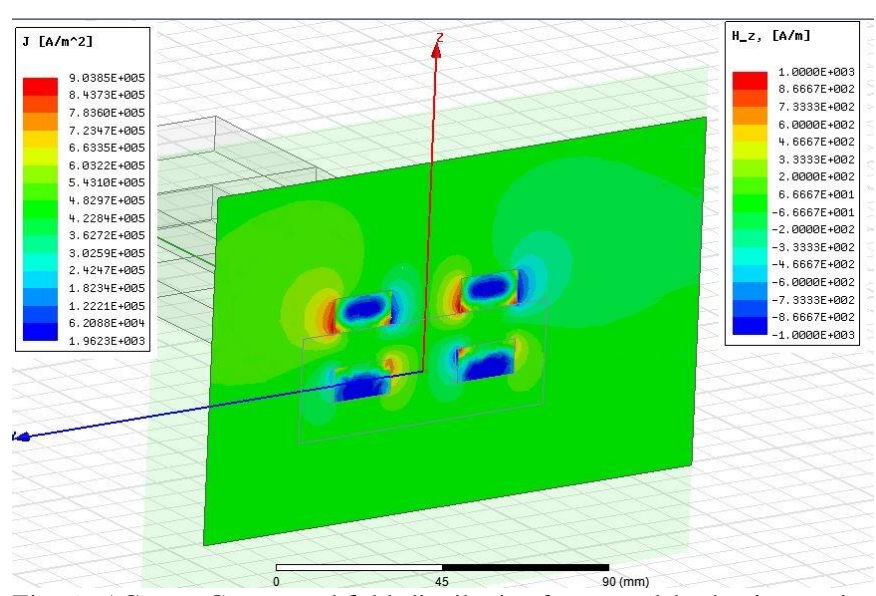

Fig. 6. AC case: Current and field distribution for external busbar in superior position in $10 \mathrm{~mm}$ distance from the measuring busbar.
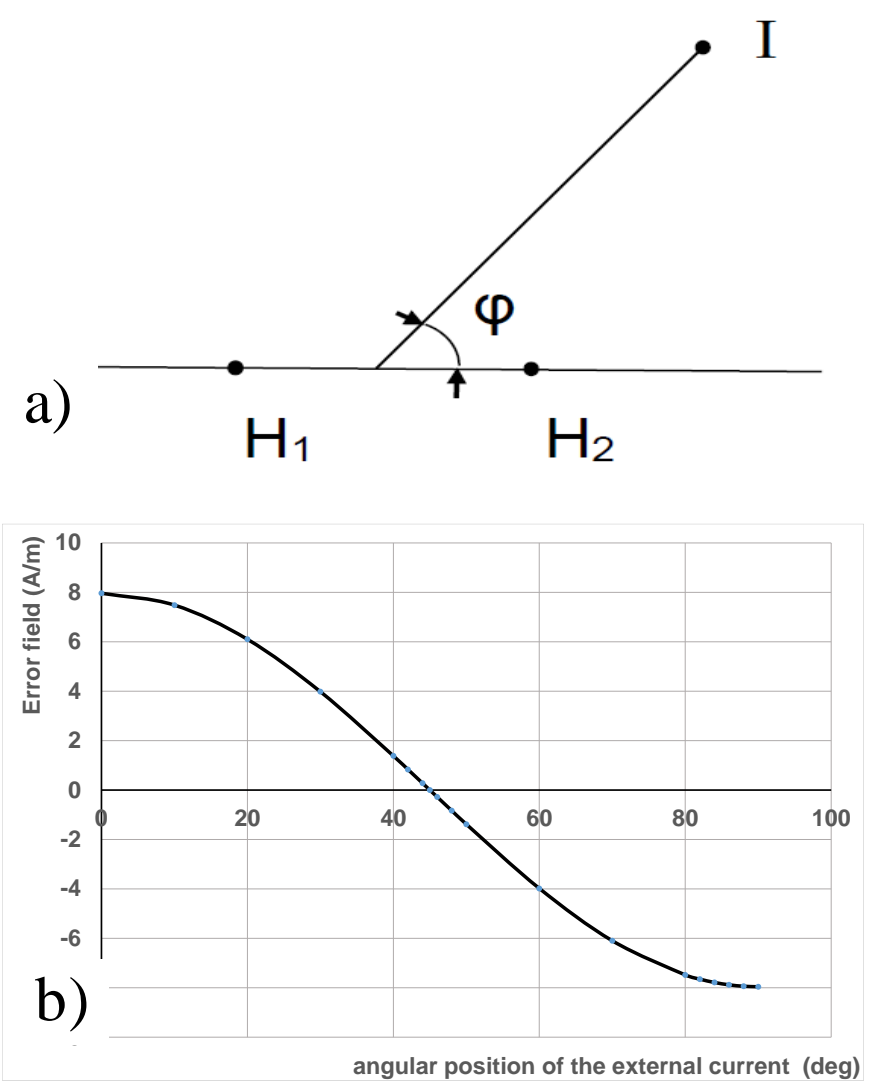

Fig. 7. External current $I$ in arbitrary angular position a) definition of the position angle, $\mathrm{b}$ ) error field as a function of angular position of an idealized $\mathrm{I}=1000 \mathrm{~A}$ in a distance of $0.1 \mathrm{~m}$

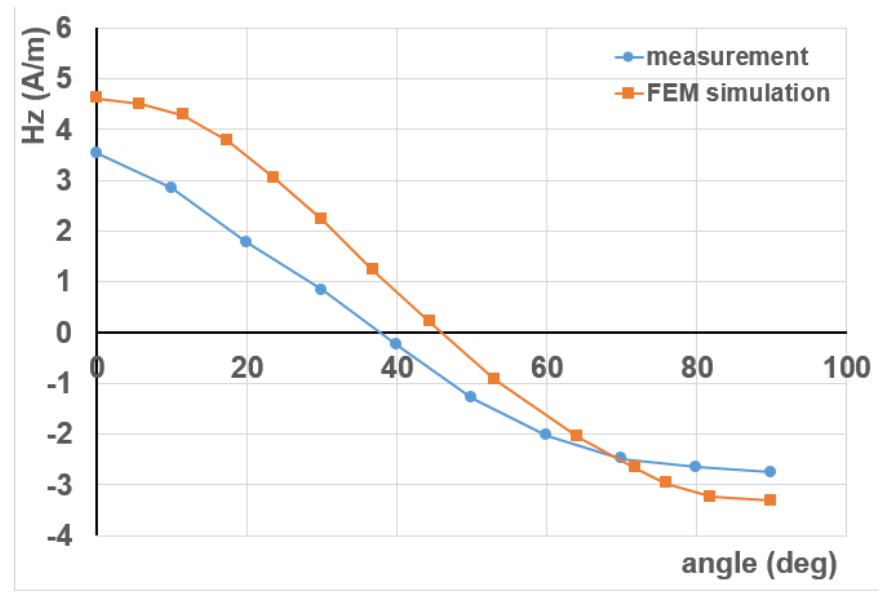

Fig. 8. Response to external dc current in busbar in arbitrary position: FEM simulation and measurement. Distance between the centers of busbars was $100 \mathrm{~mm}$, which corresponds to $40 \mathrm{~mm}$ distance between busbars 